\title{
The Influence of Increasing Age on Susceptibility of the Elderly to Tuberculosis
}

\author{
Shu-Hua Wang ${ }^{1,3}$, Bridget Carruthers ${ }^{1}$ and Joanne Turner*,1,2 \\ ${ }^{1}$ Center for Microbial Interface Biology, The Ohio State University, Columbus, OH 43210, USA \\ ${ }^{2}$ Department of Microbial Infection \& Immunity, The Ohio State University, Columbus, OH 43210, USA \\ ${ }^{3}$ Division of Infectious Diseases, Department of Internal Medicine, The Ohio State University, Columbus, OH 43210, \\ USA
}

\begin{abstract}
The elderly are susceptible to infection with the bacterial pathogen Mycobacterium tuberculosis, and to the development of active TB disease. In this review we utilize experimental data from animal models to discuss the concept that age-associated changes in immune function are a critical parameter that contributes to the increased susceptibility of the elderly to $M$. tuberculosis infection.
\end{abstract}

Keywords: Tuberculosis, aging, immunology.

Mycobacterium tuberculosis (M.tb), the bacterium that causes the disease tuberculosis (TB), infects approximately one third of the world's population and is responsible for almost 2 million deaths each year [1, 2]. TB mainly afflicts individuals that are in the prime of their life, with greater than $75 \%$ of individuals diagnosed with TB between the ages of 15 and 44 [1, 2].This can be primarily attributed to concurrent infection with the human immunodeficiency virus (HIV) [1]. However, elderly individuals (those 65 years of age and older) are also more susceptible to TB. In 2009, WHO reported that the elderly population accounted for 244,062 new smear-positive case notifications worldwide, with the majority reported from developing countries [3]. In industrialized countries, a declining trend in TB incidence has been seen in all age groups, including the elderly, however the elderly population continues to consistently have the highest age-specific incidence rates for TB each year [4].

Increased susceptibility to TB disease in the elderly has been linked to waning immune function, but can also be associated with co-morbid conditions and impaired mechanical lung function [5-7]. In this review we discuss the current state of knowledge regarding infection with M.tb and the development of TB disease in old age, with a primary focus on understanding the age associated changes in immune function that contribute to the heightened susceptibility of the elderly to develop TB. The generation and maintenance of immunity against infection with M.tb is highly complex and there are numerous stages of infection where age associated alterations in immune function can alter infection control. We will further discuss how an aging immune system can influence the establishment of primary M.tb infection as well as how increasing age can impact the long term control

*Address correspondence to this author at the Center for Microbial Interface Biology, The Ohio State University, Columbus, OH 43210, USA;

Tel: 614-292-6724; Fax: 614-292-9616; E-mail: joanne.turner@osumc.edu of an infection with M.tb. There is a paucity of immune studies on elderly individuals with M.tb infection and therefore the focus of this review is on experimental evidence that has been obtained using aged animal models.

\section{IMMUNITY TO INFECTION WITH M.tb.}

M.tb is predominantly a pulmonary pathogen, although dissemination of infection from the lung can be an important part of TB pathogenesis, and M.tb can be found in a variety of different locations within the body. The lung is, however, the primary portal of entry and residence for M.tb, and the most common location for TB disease. M.tb enters the body on aerosol droplets that are inhaled into the terminal bronchioles and alveoli of the lung, and infection is established when M.tb encounters its natural cellular niche, the alveolar macrophage [8,9]. The outcome of infection is dependent on the subsequent immune response that is generated. The relatively low transmissibility of M.tb within a population (which requires close and frequent contact) suggests that some individuals can eradicate M.tb infection. However, the exact mechanism by which this is achieved has been elusive because of the complexities of studying human immunology at the moment of infection, and because all animal models that have been tested are permissive to M.tb infection [10]. When M.tb does infect an individual, immune responses are generated that can either limit the growth of M.tb or allow bacterial growth to continue and lead to TB disease. Bacterial growth (reactivation/recrudescence) which is linked to numerous factors including HIV, malnutrition, and increasing age can occur after a period of apparent immune control $[8,9]$.

Immune control of M.tb infection is highly dependent on the generation and maintenance of T helper type 1 (TH1) immune responses [8, 9]. It has been shown in both human and murine models that T cell derived IFN- $\gamma$ as well as IL-12 and TNF produced by innate immune cells are absolutely 
critical for control of mycobacterial infection [8, 9]. Furthermore, CD4 $\mathrm{T}$ cells are essential for optimum control of infection, with CD8 $\mathrm{T}$ cells playing a less significant role. Following infection with M.tb, alveolar and infiltrating tissue macrophages become a source of IL-12 and TNF, and dendritic cells migrate to the draining lymph nodes to present mycobacterial antigens to $\mathrm{T}$ cells. A potent antigen specific CD4 and CD8 $\mathrm{T}$ cell response is generated although, unlike many other pulmonary infections, it can take up to 9 days to become measurable within the lymph node and lung tissue $[8,9]$. This delay in the generation of adaptive immunity results in significant growth of $M . t b$ within the lung before infection can be controlled (Fig. 3A). Cessation of M.tb growth occurs when antigen specific IFN- $\gamma$ secreting $\mathrm{T}$ cells are recruited to the lungs, which activate infected macrophages to employ reactive oxygen and nitrogen intermediates to kill or prevent additional replication of M.tb. Host cells form a granuloma around infected cells to wall off residual bacterium and limit the spread of infection. It is not completely clear how M.tb evades eradication but it is likely associated with its location within specific subcellular compartments that prevent its efficient detection by immune cells [11]. M.tb can remain at low levels within the lung, in a form that is termed latency or non-replicating persistence for the life-span of an individual or it may subsequently replicate leading to active TB disease $[8,9]$.

The majority of TB cases in the elderly are thought to arise from reactivation of a previously latent $M$.tb infection, where elderly individuals have been infected with M.tb when they were young, at a period of time when TB was far more prevalent. As individuals age, and age associated changes in immune function become apparent, they become more susceptible to reactivation of latent M.tb infection. Though reports of primary infection with M.tb in the elderly (development of TB disease rapidly after initial infection) are less common, elderly persons residing in long term care facilities are at increased risk of becoming infected [12, 13] and TB case rates among nursing home residents are higher than elderly persons living in the community [14]. Elderly TB patients residing in congregate settings are also capable of transmitting M.tb to others. In one report from the US, a single documented TB case within a nursing home resulted in 80 confirmed exposures among residents, staff, and visitors, and 4 new clinical TB cases [13].

The influence of increasing age on immunity to M.tb infection has primarily been studied using the aged mouse model where old mice are infected with M.tb and immune function and the capacity to control or contain infection is evaluated, a model of primary M.tb in the elderly (a newly acquired infection). Animal models of age-associated reactivation are relatively sparse in part because experimental time lines are extremely long, but also because few animal models faithfully reproduce latent $M$.tb infection making it challenging to accurately model the transition from latency to TB disease [10]. In this review we first describe what is known about primary M.tb infection in old mice, and then describe the few studies that have been performed on aging and M.tb latency and reactivation, and finally discuss the possibilities of vaccination against M.tb infection in the elderly. We close with a discussion on the challenges that are faced with the diagnosis and treatment of elderly individuals with TB. This review will be defined by three strategic questions that address topics of immunity and M.tb infection in old age.

\section{IS THE INITIAL ENCOUNTER OF M.tb WITH PUL- MONARY IMMUNE CELLS ALTERED IN OLD AGE?}

A good understanding of macrophage function in old age is lacking and the literature is often contradictory, most likely because the tissue source for macrophages can have a significant impact on cell phenotype and function [15]. In general, macrophages from elderly individuals or old mice respond to a variety of stimuli less efficiently than macrophages from young subjects and this has been linked to reduced expression and function of Toll-like receptors (TLR) [16, 17]. For M.tb infection, pulmonary macrophages are considered to be the first cell that M.tb encounters and therefore, any alteration in macrophage function with old age could have significant ramifications for how an elderly subject establishes and controls M.tb infection. In humans and young animals, M.tb interacts with macrophages via a variety of cell surface and intracellular receptors including mannose receptor (MR), complement receptors (CR), DC-SIGN, Nodlike receptors, and TLRs, each of which can impact effector function of the macrophage and dictate the intracellular fate of M.tb [11]. The capacity of macrophages to limit the growth of M.tb is dependent on the generation of a proinflammatory response including nitric oxide, TNF and IL$12[8,9]$.

Early studies of macrophage responsiveness to M.tb infection in old mice were performed using bone marrow derived macrophages, due to their abundance and ease of collection. Although these cells are not considered representative of pulmonary macrophages or cells that have aged in situ, several of the experimental findings have since been verified by studies in the lung. When bone marrow derived macrophages from elderly mice were infected with M.tb these cells were fully capable of producing a wide variety of cytokine and chemokine mRNA, indicative of a normal response to infection [18]. This included the production of iNOS mRNA for the generation of the anti-mycobacterial mediator nitric oxide. Nitrates were also detected in culture supernatants, indicating nitric oxide production, and control of M.tb intracellular growth was also equivalent between macrophages from old and young mice. In bone marrow derived macrophage cultures, the response to M.tb infection was, therefore, shown to be intact in old mice [18].

Our studies using pulmonary mouse macrophages have verified that macrophages from old mice are fully capable of producing mRNA and protein for the pro-inflammatory TH1 cytokines TNF and IL-12p40 in response to M.tb infection [19]. Furthermore, we also found no difference in the ability of pulmonary macrophages from old and young mice to take up M.tb in vitro (unpublished observations). Although cytokine production remained intact in M.tb infected macrophages from old mice, our studies revealed that the expression of several macrophage cell surface receptors were altered on the surface of pulmonary cells from old mice, compared to young. Old mice had fewer pulmonary cells that expressed $\mathrm{CD}_{11 \mathrm{c}} \mathrm{c}^{\text {hi }}$ (one of two integrin chains of CR4, and a marker 
for alveolar macrophages [20]) and more pulmonary cells that expressed CD11b ${ }^{\text {hi }}$ (one of two integrin chains of CR3 [20]) [19]. It is unclear at this time how altered CD11c/b expression, or the change in number of alveolar macrophages and monocytes within the lung can impact M.tb binding and uptake by macrophages from old mice.

Additionally, the study of TLRs demonstrated that although old mice had equivalent numbers of pulmonary macrophages that could express the pattern recognition receptors TLR2 and TLR9 as young mice, the relative expression (density) of TLR2 on the cell surface was significantly increased [19]. This change in TLR2 surface density may be a compensatory mechanism for altered function of TLR2 in old age. Indeed, the study of TLR gene disrupted (KO) mice has shown that for young mice TLR2 recognition of M.tb is essential for the secretion of IL-12p40 and TNF by macrophages [21] yet, in contrast, pulmonary macrophages isolated from old TLR2 KO mice were fully capable of secreting IL-12p40 and TNF in response to M.tb [19]. TLR2, although present on pulmonary macrophages from old mice at an increased density, is clearly dispensable for recognition of M.tb. Furthermore, antibody blocking or inhibitors showed that other TLRs known to interact with M.tb (TLR4 and TLR9) were only partially able to compensate for the TLR2 independent secretion of pro-inflammatory cytokines in response to M.tb that was observed in macrophage cultures from old mice [19]. Therefore, in old age pulmonary macrophages diversify and become capable of recognizing M.tb via different, as yet uncharacterized, receptors that can mediate the secretion of pro-inflammatory cytokines [19]. These findings may have direct application to the development of new vaccines that utilize TLR agonists to boost the immune response, and indicate that TLR2 agonists may not be as effective in the elderly as they are in younger individuals.

Differential recognition of M.tb by cell surface receptors may also impact the intracellular fate of bacteria and the subsequent generation of an immune response. In addition to receptor mediated recognition of M.tb mediated by TLRs, M.tb also interacts with phagocytic receptors. The type of receptor that facilitates internalization of $M . t b$ can also alter infection outcome (survival versus killing of M.tb). In humans, MR mediated uptake of M.tb directs the bacteria to an intracellular compartment that is refractory to cell killing and promotes an anti-inflammatory state of the macrophage, [11]. Although pulmonary macrophages from old mice are known to have altered expression of the CR chains CD11b and CD11c on the surface (more CR3 and less CR4), there is very little known about the expression and functional capacity of other cell surface or intracellular pathogen recognition receptors on macrophages in old age. Pulmonary macrophages also serve a critical role in communicating with the adaptive immune system via the presentation of M.tb antigens to CD4 and CD8 T cells. MHCII expression on antigen presenting cells can be variable in old age [22, 23], but we have found that macrophages from the lungs of old mice express either equivalent or increased levels of MHCII (Fig. 1A) and co-stimulatory molecules (unpublished observations). In addition, macrophages from old mice were also fully capable of presenting M.tb antigen to CD4 T cells, determined using T cell lines that are specific for an M.tb protein (Fig. 1B). Therefore, these preliminary studies indicate that the capacity of macrophages from old mice to present antigen to the adaptive arm of the immune system appears to be intact, however additional studies are necessary to confirm this in vivo.

Innate immunity, in response to primary infection with $M . t b$, appears to be functional in old age, at least with regard to macrophage function. Macrophages from old mice can secrete pro-inflammatory cytokines in response to infection and appear to control M.tb infection similarly to macrophages from young mice. What is different in old age is the usage of TLRs to recognize M.tb infection and generate cytokine production, with macrophages from old mice being fully capable of responding to M.tb infection in the absence of TLR2. The consequences of this altered pathogen recognition during initial infection are yet to be recognized. Furthermore, in contrast to the studies of macrophage function in response to primary M.tb infection in old age, no studies have been performed to determine how macrophages maintain control of M.tb during a chronic or latent infection.

\section{IS ADAPTIVE IMMUNITY TO M.tb INFECTION DE-}
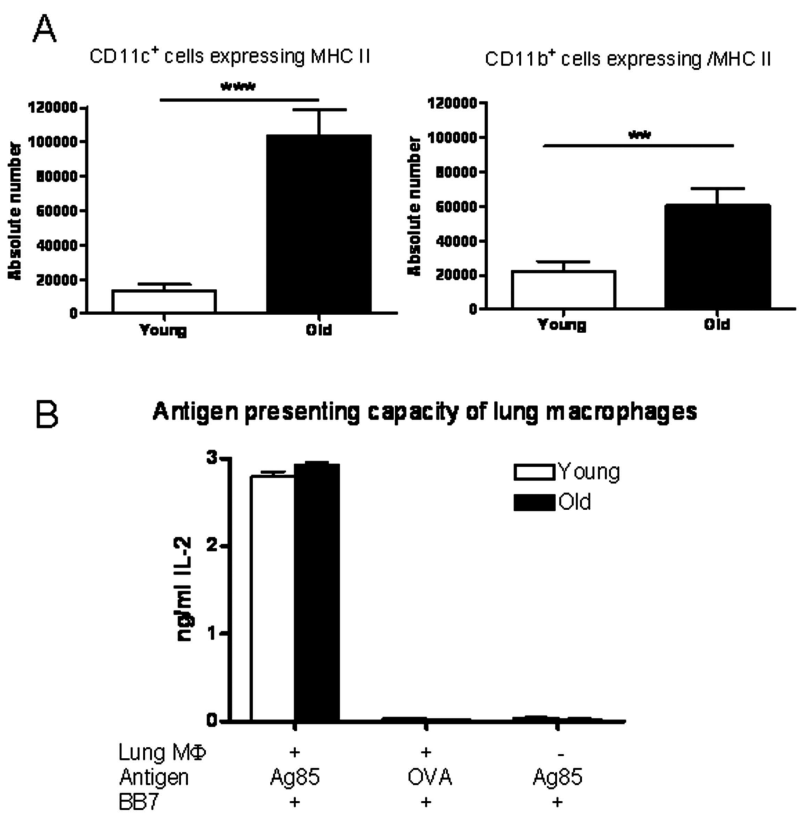

Fig. (1). Properties of pulmonary macrophages from old mice.

(A) Pulmonary macrophages were isolated from old (18 month) or young (3 month) mice and labeled with fluorescent antibodies specific for CD11b, CD11c, and MHCII. Cells were analyzed by flow cytometry and reported as the absolute number of CD11b or CD11c cells that express MHCII. Cells from old mice also had increased fluorescence intensity indicating increased abundance of MHCII in addition to increased numbers of MHCII positive cells (not shown). Data are from 5 mice from each group and representative of 3 independent experiments. Statistical significance determined by Student's $t$ test; ${ }^{* *} \mathrm{p}<0.01,{ }^{* * *} \mathrm{p}<0.001$. (B) Pulmonary macrophages were isolated from old (18 month) or young (3 month) mice and incubated overnight with the M.tb antigen Ag85 or ovalbumin (OVA). Following a wash step macrophages were co-cultured with clone BB7, specific for Ag85 peptide, for 24 hours and IL-2 detected in supernatants. Data are representative of 2 experiments. 


\section{FECTIVE IN OLD AGE?}

Several studies have demonstrated that increasing age is associated with alterations in the adaptive immune system, leading to suboptimal $\mathrm{T}$ and $\mathrm{B}$ cell function that can impact responses to vaccination or infection [24-26]. Protective immunity to infection with M.tb is highly dependent on the generation of antigen specific T cell mediated immunity [8], although B cells also play an important role [27]. With regard to studies of M.tb infection in old age, the role of B cells and antibody have not been addressed. Studies of primary M.tb infection of old mice have demonstrated that in old age, $\mathrm{T}$ cell function can be divided into two different clusters; cells expressing properties of the innate immune system and cells expressing properties of the adaptive immune system.

\section{INNATE LYMPHOCYTE FUNCTION IN PRIMARY M.tb INFECTION}

We have previously identified an early resistance mechanism to M.tb infection within the lungs of old mice that is mediated by CD8 $\mathrm{T}$ cells and occurs in an antigen independent manner [28]. The term 'early resistance' was coined to reflect the transient control of M.tb growth within the lungs of old mice relative to young mice, that was evident for approximately 2-3 weeks of infection, after which growth increased to levels that often exceeded that seen in young mice [28]. Therefore, pulmonary CD8 T cells from old mice are capable of responding to M.tb infection via an innate immune mechanism. This early resistance to M.tb infection was first described by Cooper et al, one of the first groups to use low dose aerosol M.tb infection of old mice, who investigated the role of IL-12 in boosting immune function in old age [29]. Despite these previous observations, the early resistance phenotype was not studied in detail or linked to CD8 $\mathrm{T}$ cells for several more years. The first indication that CD8 $\mathrm{T}$ cells participated in the expression of early resistance was when old CD8 KO mice failed to limit early M.tb growth in the lungs [28]. A population of highly activated IFN- $\gamma$ producing CD8 T cells were also identified within the lungs of naïve as well as M.tb infected old mice [28]. Additional studies using M.tb infected IFN KO old mice indicated an important role for IFN- $\gamma$, but not IFN type I, in mediating early resistance [30].

In vitro studies have elucidated a mechanism for the IFN$\gamma / \mathrm{CD} 8 \mathrm{~T}$ cell mediated early resistance to infection with $M . t b$ that is observed in old mice. In addition to secreting IFN- $\gamma$ in response to $\mathrm{T}$ cell receptor cross-linking, CD8 T cells from the lungs of old mice were also responsive to the TH1 cytokines IL-12, IL-18, and IL-2 [31]. In combination, these three cytokines were capable of stimulating a large proportion of pulmonary CD8 $\mathrm{T}$ cells from old mice to produce IFN- $\gamma$, a mechanism that has also been described for a minor subset of CD8 $\mathrm{T}$ cells in young mice [32]. This responsiveness was facilitated by the increased expression of TH1 cytokine receptors on the cell surface of pulmonary CD8 T cells from old mice [31]. Where pulmonary CD8 T cells from old mice differed functionally from young however, was in their capacity to produce IFN- $\gamma$ in response to one single cytokine, IL-12p70. Reactivity was exclusive for IL12 p70 and not other members of the IL-12 family (p40 monomer, p40 homodimer, or p35), and was facilitated by enhanced IL-12p70 signaling pathways [31]. It was also shown that old mice had increased proportions of activated (CD44 ${ }^{\mathrm{hi}}$ ) CD8 T cells that could phosphorylate STAT4 in response to IL-12p70, which led to the subsequent downstream production of IFN- $\gamma$ production [33]. The increased STAT4 phosphorylation observed within pulmonary CD8 T cells from old mice was linked in part to the enhanced endogenous activity of the phosphatase inhibitor SET, which serves to inhibit the normal dephosphorylation activity of PP2A (a serine threonine phosphatase that dephosphorylates STAT4) [33]. In the presence of enhanced SET activity, and therefore reduced PP2A activity, IL-12 signaling could take place at greater or enhanced levels in CD8 T cells from old mice (depicted in Fig. 2). The exact mechanism and relevance for elevated SET activity has yet to be elucidated.

Evidence that CD8 T cells might respond directly to IL12 in vivo during M.tb infection was demonstrated with the measurement of increased IFN- $\gamma$ mRNA from pulmonary CD8 $\mathrm{T}$ cells from old mice in response to lung cell culture with M.tb, or during co-cultured with purified M.tb infected antigen presenting cells [34]. Indeed, supernatants from M.tb infected cells could stimulate pulmonary CD8 T cells from old mice to secrete IFN- $\gamma$ directly, an effect which could be abrogated in the presence of anti-IL-12. IFN- $\gamma$ production was also prevented if antigen presenting cells were deficient in IL-12p35 but not if cells were devoid of $\beta 2 \mathrm{~m}$ (lacking MHC class I), demonstrating an IL-12 dependent and antigen independent mechanism of IFN- $\gamma$ stimulation [34]. Perhaps the most significant evidence that pulmonary CD8 T cells from old mice could respond to IL-12 production in vivo in response to M.tb was the finding that elevated levels of IL-12p40 and IFN- $\gamma$ mRNA or protein could be measured within the lungs of old mice infected with M.tb as early as 5 days post infection [31]. Furthermore, the isolation of CD8 T cells directly from the lungs of old mice during M.tb infection showed that IFN- $\gamma$ and SET mRNA could be detected within this specific $\mathrm{T}$ cell subset as early as 8 days post infection [34]. Pulmonary CD8 T cells from the lungs of M.tb infected old mice were, therefore, a dominant source of IFN$\gamma$ in vivo, and also showed evidence of similar SET regulation as was observed in vitro. Based upon the elevated levels of IL-12 within the lungs of old mice infected with M.tb, we can anticipate that CD8 T cells are also capable of responding to infection in an antigen independent manner in vivo, leading to IFN- $\gamma$ secretion and enhanced early control of $M . t b$ infection. We can also speculate that the improved outcome observed in old M.tb infected mice that were treated with IL-12 as noted by Cooper et al [29] may have been a consequence of boosting innate CD8 T cells within the lung, in addition to the improved CD4 $\mathrm{T}$ cell responses that were reported.

\section{ADAPTIVE LYMPHOCYTE FUNCTION IN PRIMA- RY M.tb INFECTION}

Studies of adaptive immunity have, for the most part, been performed using the primary M.tb infection model of old mice. There have also been a small number of studies of $\mathrm{T}$ cell function during chronic M.tb infection that have taken the infection model out to a period of time that can be impacted by age associated immune changes. In primary infec- 


\section{YOUNG}

\section{OLD}

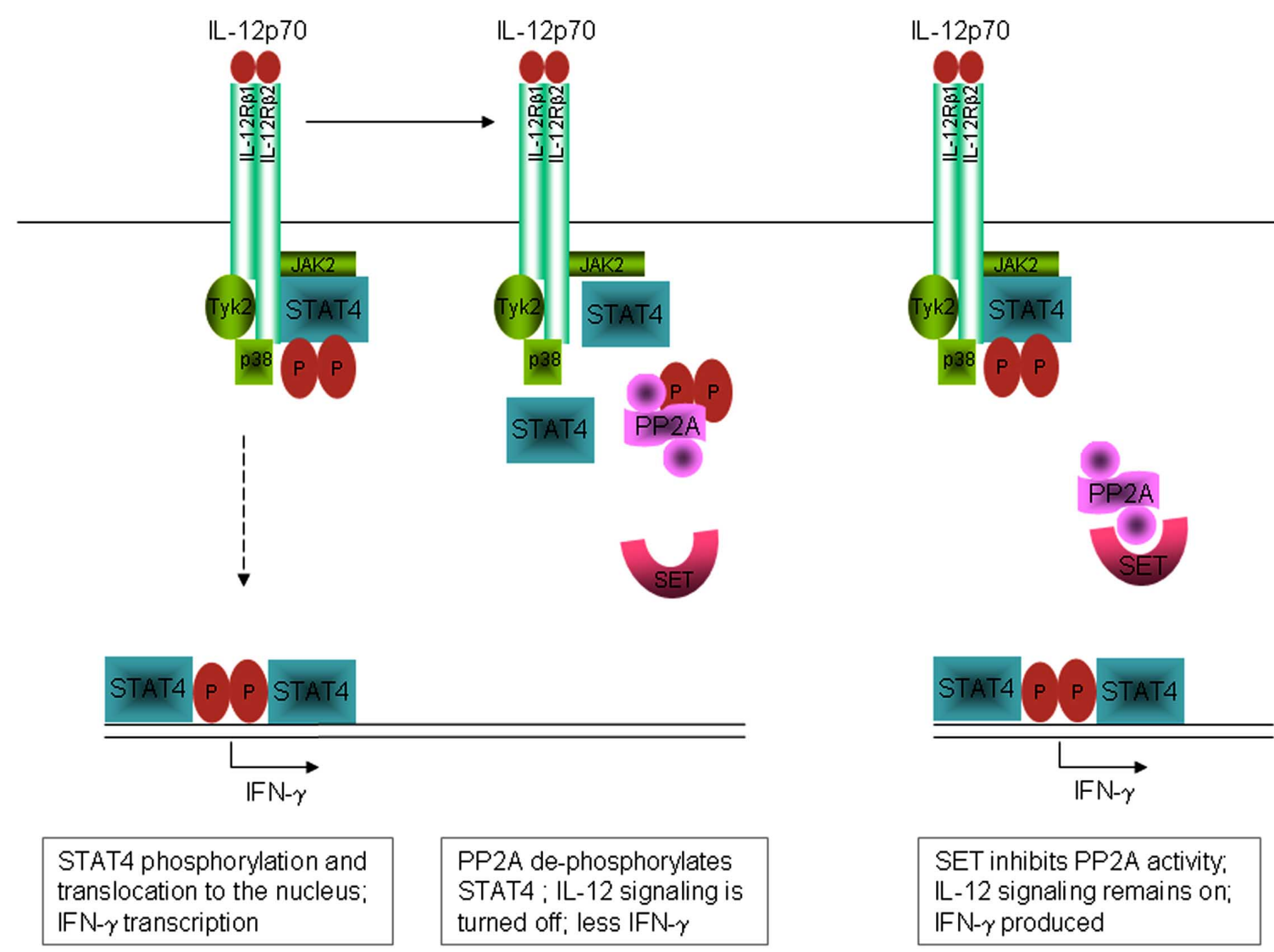

Fig. (2). Regulation of IL-12 signaling in CD8 T cells from old mice.

IL-12 signaling occurs when IL-12p70 binds to the IL12R $\beta 1 / \beta 2$ receptor, leading to recruitment of Jak2/Tyk2/p38 and phosphorylation and dimerization of STAT4. Phosphorylated STAT4 translocates to the nucleus and facilitates the transcription of IFN- $\gamma$. In CD8 T cells from young mice, IL-12 signaling is regulated via the dephosphorylation of STAT4 by PP2A. In CD8 T cells from old mice, the function of PP2A is inhibited by SET, leading to continued IL-12 signaling and IFN- $\gamma$ production.

tion, old mice are considered to be more susceptible to M.tb infection than young mice. This was first demonstrated using intravenous M.tb infection which clearly showed that mice exceeding 20 months of age were highly susceptible to M.tb infection and failed to show any evidence of control of M.tb growth within the lung or peripheral organs [35]. Adoptive transfer studies also indicated that old mice had deficiencies within the T cell compartment, because adoptive transfer of immune $\mathrm{T}$ cells from young mice were fully capable of imparting protection on older mice, whereas the reciprocal experiment provided only limited protection [36]. These studies clearly identified cells of the adaptive immune system ( $T$ cells) as the population that led to impaired M.tb infection control in old mice. The mechanism for this altered $\mathrm{T}$ cell function in old mice during M.tb infection has not been fully elucidated however, studies also showed that both CD4 and CD8 $\mathrm{T}$ cells were slow to accumulate in the spleen following an intravenous infection with M.tb [37]. In contrast, $\gamma \delta$ T cells accumulated at a similar rate to young M.tb infected mice indicating no deficiencies in this $\mathrm{T}$ cells population [37], a finding that has also been verified using aged $\gamma \delta \mathrm{KO}$ mice [38]. Although the delayed accumulation of T cells and the increased growth of $M . t b$ in old mice provided evidence for a deficiency in $\mathrm{T}$ cell function, the study of antigen specific $\mathrm{T}$ cell responses revealed that old M.tb infected mice were fully capable of generating M.tb specific CD4 T cells in the spleen, but their responsiveness was delayed compared to young mice [37]. With additional studies demonstrating altered integrin expression on splenic CD4 and CD8 T cells from old mice, both prior to and following intravenous M.tb infection [39], it was hypothesized that the delay in $\mathrm{T}$ cell accumulation to the spleen may reflect poor $\mathrm{T}$ cell migration to this organ [37]. Therefore, studies using the systemic intravenous M.tb infection model nicely demonstrate that, at least in the spleen, old mice were more susceptible to infection with $M . t b$, and that this was associated with a delay in the generation of M.tb specific CD4 T cells. This delay would likely allow M.tb to grow unrestricted for a longer period of time, reaching greater numbers within primary organs before immune control. We can speculate that this results in pathological tissue damage that precedes and contributes to morbidity and mortality of old mice, although this has yet to be verified. 
The intravenous infection model for M.tb has been a valuable tool for the study of susceptibility to M.tb infection and immune responses in old age however, the intravenous delivery route deposits high numbers of M.tb to the peripheral organs (primarily spleen and liver) and seeds the lung via the bloodstream. In contrast, in man M.tb is most commonly transmitted via the respiratory route, delivering a low inoculum of M.tb bacilli into the pulmonary tract. Therefore, the low dose aerosol model of M.tb infection, where approximately 70 M.tb bacilli are delivered to the lung in droplet aerosol, is considered a more realistic model of M.tb infection in man. This has been the model system most favored for the study of early innate immune events within the lungs of old mice in response to M.tb infection however, publication of studies with regard to adaptive immune function using this model are relatively sparse.

In young mice infected via the respiratory route, adaptive immunity is generated within the first 1-2 weeks of infection $[8,40]$. Stabilization of the pulmonary bacterial load is evident by the third week of infection, which is highly associated with the presence of antigen specific CD4 $\mathrm{T}$ cell in the lung (Fig. 3B). Interestingly, infection of old mice via the respiratory route reveals that old mice can accumulate similar numbers of $\mathrm{T}$ cells within the lung as young mice, with only a modest and non-significant delay in the accumulation of CD4 T cells [28]. Normal accumulations of CD8 T cells were also apparent in the lung [28]. We have also found that the number of CD8 T cells that label positive for a M.tb peptide tetramer is also unaltered in old mice (unpublished observations). Furthermore, the detection of both CD4 and CD8 $\mathrm{T}$ cells within the lung that were capable of secreting the protective cytokine IFN- $\gamma$ (in response to TCR crosslinking) at the peak of protective immunity was not significantly different between young and old mice [28]. We have recently measured the frequency of $M . t b$ antigen specific IFN- $\gamma$ secreting CD4 T cells in the draining lymph nodes and lungs of old mice infected with M.tb via the pulmonary route, using ELISPOT, and also found no significant differ- ences between young and old mice (Fig. 3B, C). In fact, responses in the draining lymph nodes appeared accelerated in old mice, indicting that old mice were fully capable of generating an adaptive immune response if M.tb was delivered to the lungs. Therefore, when old and young mice are infected with M.tb via the natural respiratory route, there is little evidence for an altered $\mathrm{T}$ cell response in old age. Despite this, the control of M.tb within the lungs of old mice appears to be altered, with increased M.tb growth within the lung as infection progresses beyond 35 days (Fig. 3A) [28, 29]. Therefore it will be important to determine whether antigen specific $\mathrm{T}$ cells are functional in vivo during infection, and if they are not, identify factors that may impair their function. In this regard, studies have shown that aged mice have increased numbers of IL-10 producing phagocytes in the lung [41] that can actively suppress the effector function of antigen specific CD4 T cells in vivo [42].

The generation and maintenance of antigen specific CD4 $\mathrm{T}$ cells is critical for the control of M.tb infection yet our knowledge of how the aged adaptive immune system functions in vivo during M.tb infection is lacking. Studies appear to be contradictory in nature, with intravenous infection models indicating that the accumulation of antigen specific $\mathrm{T}$ cell are delayed in old age, perhaps as a consequence of poor cell migration facilitated by altered adhesion molecule expression. In contrast, studies using the respiratory route of infection indicate that $\mathrm{T}$ cell function is intact, yet old mice do remain more susceptible to M.tb infection than young mice. Additional studies are necessary to fully understand the $\mathrm{T}$ cell intrinsic (such as cellular senescence) and extrinsic properties (IL-10 production for example) that contribute to the increased susceptibility of the elderly to infection with M.tb.

\section{The Influence of Increasing Age on Control of Chron- ic/Latent M.tb Infection}

In man, the most significant cause of TB is the reactivation of a previously latent M.tb infection, caused by a variety
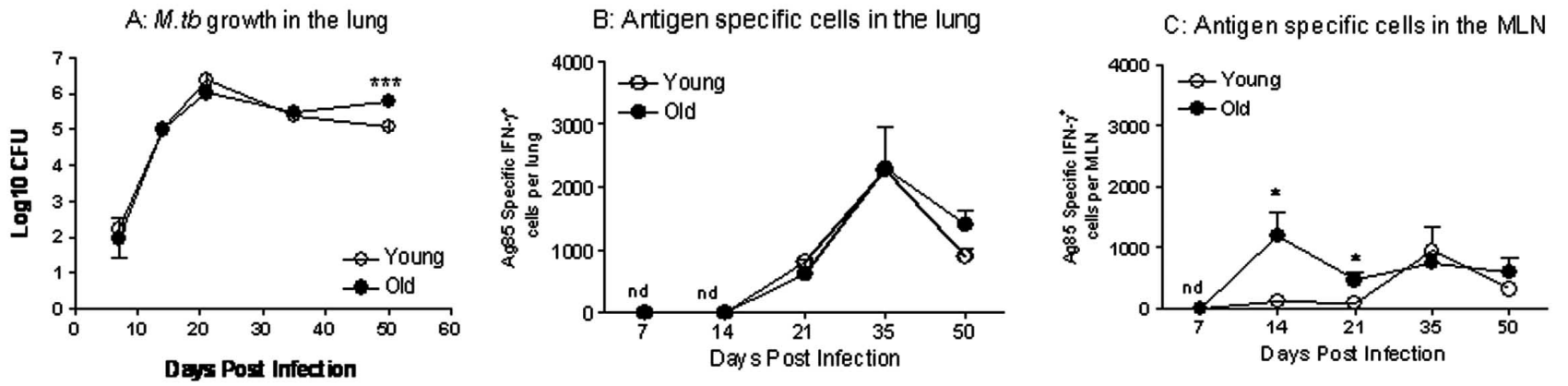

Fig. (3). Antigen specific CD4 T cell responses during M.tb infection.

(A) Old (18 month) and young (3 month) mice were infected with M.tb using a Glas-col inhalation exposure device calibrated to deliver 50100 colony forming units (CFU) to each individual mouse. At designated time points lungs were collected and homogenized, and plated onto 7H11 agar plates to determine CFU. Statistical significance was determined using Student's $t$ test: ***, p<0.001. Data are mean and SEM from 2 independent experiments using $n=5 /$ mice per group at each time point. (B, C) Single cell suspensions were obtained at various time points post infection from lung (B) or MLN (C) of old (18 month) and young (3 month) M.tb infected mice. CD4 cells were purified using magnetic beads and adjusted to 1 × $10^{6}$ cells/ml. CD4 cells were serially diluted and incubated with Ag85 pulsed, paraformaldehyde fixed, DCs (derived from young mice). Cells were cultured on ELISPOT plates coated with anti-IFN- $\gamma$ for 3 days. Ag85 specific IFN- $\gamma$ spots were enumerated using an Immunospot Analyzer (CTL). Data are mean and SEM and representative of 5 mice per time point. nd= not detected. Statistical significance was determined by Student's $t$ test: *, $\mathrm{p}<0.05, * *, \mathrm{p}<0.01$. 
of factor such as malnutrition, HIV infection, or poverty, all of which can be linked in some way to immune deficiency. Reactivation of latent M.tb infection is also thought to be the main form of TB in the elderly, driven by age associated changes in immune function [5]. The study of reactivation TB in any context is challenged by the lack of suitable animal models that can develop latent M.tb infection, the form that remains quiescent in man until subsequent reactivation. With the exception of non-human primates all animal models tested have failed to establish latency, and instead develop chronic progressive disease [10]. For aging studies the nonhuman primate is a challenging model due to its extended lifespan and therefore, despite its limitations, the chronic mouse model has been frequently used to study the influence of aging on control of M.tb infection (Fig. 4A, B). In the chronic model, the initially stable bacterial load within the lung increases to levels associated with morbidity and mortality as mice increase in age [43]. In 1988 Orme proposed the mouse as a model of age associated recrudescence of latent M.tb infection [44]. His studies showed that mice infected with a very low dose of M.tb (undetectable at initial determination) when young could control a chronic infection for at least 18 months, after which mice died approximately

\section{A: The chronic model of $M t b$ infection}

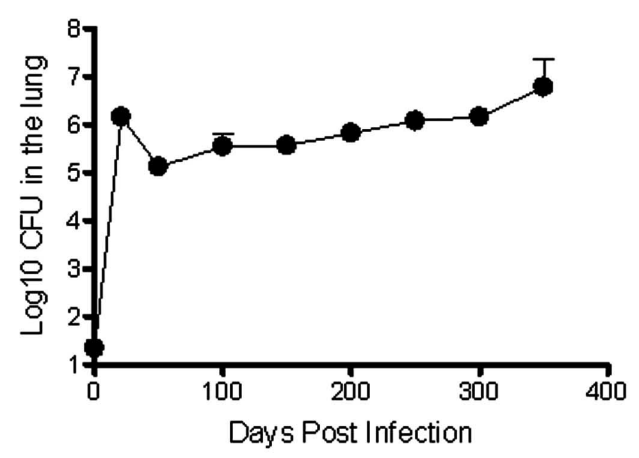

C: PD1 expression on T cells in lung

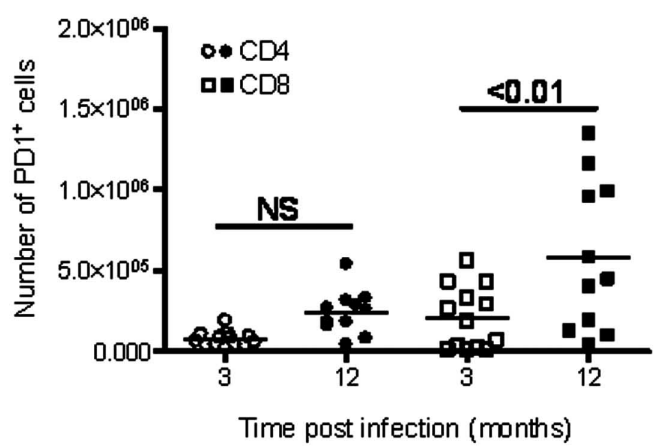

6 months earlier than non-infected control mice. Death was associated with increasing M.tb growth within the lungs [44]. The hypothesis generated was that recrudescence of $M . t b$ infection in older mice was linked to an age-related failure to generate or maintain a memory recall response [44]. An alternative hypothesis is that chronic M.tb infection leads to constant $\mathrm{T}$ cell activation and eventual exhaustion [45], and the eventual loss of functional protective immune cells. Our studies have determined that CD8 $\mathrm{T}$ cells isolated from the lungs of mice that have been chronically infected with M.tb for 12 months express the immune exhaustion and senescence markers PD1 and KLRG1 (Fig. 4C, D), indicating that CD8 $\mathrm{T}$ cells may become altered in the presence of ongoing stimulation, which could result in an age-associated loss of immune control of M.tb infection.

Granuloma formation is also an integral component of $M . t b$ infection control and studies using the chronic M.tb infection model have shown that progressive granuloma degeneration within the lung, with pulmonary damage that becomes extensive as lesions coalesced and become necrotic, is associated with increased M.tb growth [43]. Although increasing age and associated immune changes may play a role in recrudescence of $M . t b$ infection, for the mouse model is it

\section{B: Survival of $\boldsymbol{M} t \boldsymbol{b}$ infected mice}
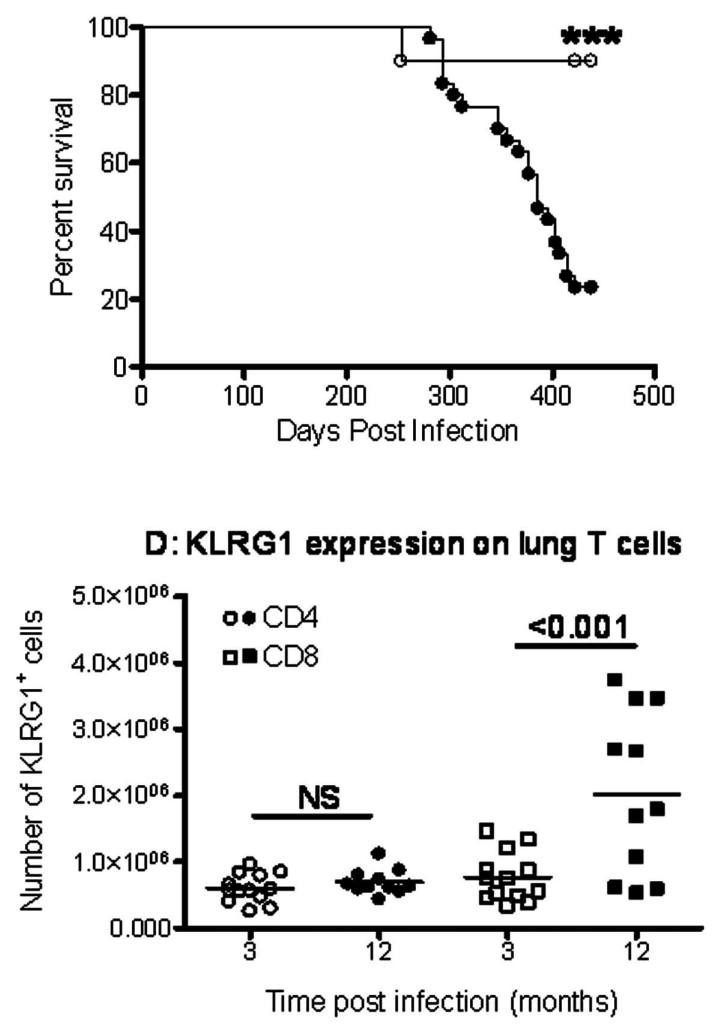

Fig. (4). The chronic M.tb infection model.

3 month old C57BL/6 mice (solid circles) were infected with M.tb Erdman. Non-infected controls (open circles) were maintained in the same facility under identical housing conditions. (A) CFU detected at designated time points from 5 mice and expressed as Log10 CFU/organ. (B) Mice that showed indicators of distress (huddling, rapid breathing, isolation from cage-mates) were euthanized. Statistical analysis by Logrank; *** $\mathrm{p}<0.0001$. (C,D) Lung cells from mice that had been infected with M.tb for 3 or 12 months were labeled with anti-CD4, CD8, CD3, KLRG1, and PD1 and analyzed by flow cytometry. Data are presented as the absolute number of CD4 and CD8 T cells in the lung that expressed PD1 (C) or KLRG1 (D). Data are combined from 4 independent experiments with a total of 9-13 mice. Statistical analysis; oneway ANOVA with Tukey's post test. 
almost impossible to clearly dissociate and study the role of age-associated immune function independent of the progressive tissue damage driven by chronic M.tb infection. A more suitable model for latent M.tb is necessary to fully understand how increasing age can impact the long term control of latent M.tb infection.

Reactivation TB is considered to be the most common form of TB in the elderly and yet there is very little experimental data available to discern how increasing age can impact control of M.tb infection. Studies of chronic M.tb infection in the mouse indicate that failure to control infection may be linked to poor memory responses or $\mathrm{T}$ cell senescence, yet how these directly impact and contribute to the loss of compact granuloma formation and integrity is as yet unclear. Furthermore, the relevance of using the chronic M.tb infection model for the study of latent M.tb infection has to be addressed and until more realistic models can be generated it is likely that our understanding of age-associated reactivation will remain limited.

\section{IS SUCCESSFUL VACCINATION OF THE ELDERLY AGAINST M.tb INFECTION ACHIEVABLE?}

The current vaccine for protection against M.tb infection is $M$. bovis Bacille Calmette Guerin (BCG), an attenuated $M$. bovis strain of mycobacteria. BCG has been delivered extensively throughout the world, and provides protection against childhood TB, particularly for TB meningitis. There is little evidence that BCG vaccination can provide life-long immunity and studies in animal models indicate that the protective efficacy of BCG wanes fairly quickly over time [46-48]. There are, however, promising experimental data to indicate that booster vaccination for the elderly may provide some protection against infection with M.tb. Studies have shown that boosting BCG vaccinated mice (vaccinated when young) with a dominant and immunogenic M.tb protein in adjuvant in mid-life (9-12 months of age) can provide significant protection against challenge with virulent M.tb infection at 20 months of age [48]. Protection could also be afforded to aged guinea pigs with a booster vaccination of BCG [46], which was associated with the generation of a robust antigen specific IFN- $\gamma$ response. Furthermore, intraperitoneal delivery of BCG to naïve old mice was shown to provide some protection from subsequent challenge with M.tb [49] indicating that vaccination when old, or booster vaccination in old age, may be successful strategies to protect the elderly against primary M.tb infection. Given that a large proportion of the world has previously been BCG vaccinated in childhood, it is reassuring to observe that a boosting strategy can be successful in old age.

\section{CLINICAL CHALLENGES FOR THE DIAGNOSIS AND TREATMENT OF TB IN THE ELDERLY}

There is a distinct lack of experimental data from elderly individuals infected with (latent) M.tb, or with active TB disease, and therefore information about susceptibility to infection has relied heavily on studies performed in aged animals. It is unclear at this time how we can extrapolate experimental animal studies to man, and comparative studies are absolutely necessary as we moved forward and attempt to understand why the elderly are more susceptible to TB.
There are considerable challenges with diagnosing and treating elderly subjects with TB disease.

\section{Diagnosing TB in the Elderly}

The diagnosis of active TB disease is based on clinical, epidemiological, radiological, and bacteriological data. Inadequate sputum production in the elderly can lead to difficulties obtaining a specimen for acid-fast bacilli smear and culture to aid in diagnosis [50]. Furthermore, interpretation of clinical and radiological parameters can also be complicated in the elderly. There is a general perception that elderly TB patients present with nonspecific or fewer classic TB symptoms however, a meta-analysis has shown no significant differences between old and young TB patients with regard to prevalence of cough, sputum production, weight loss, fatigue/malaise [50]. Chest radiograph, however, may vary between young and elderly TB patients. In the elderly, chest radiograph abnormalities due to TB may either be absent or visualized in the mid to lower zones rather than upper lung fields, often leading to a misdiagnosis of bronchitis or pneumonia [51]. Pleural TB and advanced pulmonary disease are also more likely to occur in the elderly [52, 53]. An increased incidence of smoking and cancer can also contribute to misdiagnosis of malignancies on chest radiograph. Studies are mixed as to whether elderly TB patients experience a longer time from first suspicious complaint to actual diagnosis of TB when compared to younger patients $[50,54,55]$ however, the consequences of delay in diagnosis can result in increased opportunity for TB transmission as well as substantial disease progression before medical intervention is initiated, which may lead to increased TB mortality in the elderly.

Once active TB is diagnosed in an elderly person, differentiation between recent transmission and reactivation of past infection is critical for public health purposes. Active $\mathrm{TB}$, due to recent transmission, requires contact investigation to identify the source case, in addition to identifying contacts that were exposed in order to control the spread of infection. Molecular fingerprinting of M.tb isolates can identify clusters of identical genotypes that are presumed to be linked by recent transmission events, which is helpful for initiating an expanded contact investigation to identify additional cases [56]. For example, in one study several health care workers in a nursing home had tuberculin skin test (TST) conversions, prompting an evaluation using molecular genotyping and epidemiological analyses. Ultimately four cases of active TB were molecularly linked to one nursing home patient [13].

Although the TST has been the traditional screening method for latent M.tb infection there have been reports of poor TST reactivity in the elderly $[57,58]$. A two-step skin test is recommended for patients entering nursing homes, which may identify increased numbers of individuals who have been exposed to M.tb, and the newer IFN- $\gamma$ release assays (IGRAs) also offer several advantages for diagnosis of elderly individuals exposed to M.tb [59], including a positive control to identify potentially anergic patients. Though IGRA may be more specific than TST, limited data on the reliability of this new test are available for elderly individuals with active TB disease [60, 61]. 


\section{TB Treatment for Elderly Individuals}

TB treatment principles and drug regimens are the same for elderly and younger populations [62] where initiation of a four-drug regimen of rifampin (RIF), isoniazid (INH), pyrazinamid (PZA), and ethambutol (EMB) is administered until drug susceptibility testing is completed. Because treatment duration is a minimum of 6 months for drug susceptible pulmonary TB, and 9 to 12 months for TB meningitis or TB osteomyelitis, elderly TB patients that are on multiple other medications may face problematic drug interactions. RIF is a potent inducer of microsomal liver enzymes and increases the metabolism of several drugs that are metabolized by the liver, such as coumadin (leading to decreased anticoagulation), sulfonylureas for diabetes (leading to decreased blood sugar control), and beta blockers and calcium channel blockers (leading to decreased blood pressure control) [62]. Treatment monitoring is necessary for elderly patients, because many studies indicate a higher incidence of adverse reactions to TB therapies in this population [63-65]. In one study, major drug toxicity required an alteration in therapy in $40 \%$ of patients over age 65 [66]. First-line TB medications can also cause hepatoxicity. Studies have shown that during treatment for latent M.tb infection, $4.5 \%$ of elderly patient experienced INH-induced hepatitis and 23\% experienced neutropenia [67]. Directly Observed Therapy (DOT) is also particularly important for monitoring side effects and maintaining treatment adherence in elderly patients with dementia or confusion due to other diminished mental capacities.

The significant complications of diagnosing and treating elderly individuals with TB make the development of a new vaccine that is effective against M.tb infection in the elderly highly desirable. Although studies in animal models have shown promising protection results for mid-life boosting vaccines, as well as vaccination against M.tb in older animals [46, 48, 49], additional studies are necessary to fully validate this as an approach to protect the elderly against M.tb infection. Furthermore, there is a critical need to evaluate immune function in elderly individuals that have latent M.tb infection or TB disease so that we can generate a comprehensive understanding of how the elderly respond to M.tb infection. This information will not only facilitate the design of vaccines against M.tb but will also enable researchers to choose the most appropriate animals models to study this challenging infectious disease.

\section{ACKNOWLEDGEMENTS}

This work was supported by a Julie Martin Mid-Career Award to JT from The American Federation for Aging Research (AFAR).

\section{CONFLICT OF INTEREST}

None declared.

\section{REFERENCES}

[1] Corbett EL, Watt CJ, Walker N, et al. The growing burden of tuberculosis: global trends and interactions with the HIV epidemic. Arch Intern Med 2003; 163(9): 1009-21.

[2] Kochi A. The global tuberculosis situation and the new control strategy of the World Health Organization. Tubercle 1991; 72(1): $1-6$.
[3] WHO. Global tuberculosis control 2010. 2010.

[4] CDC. Reported tuberculosis in the United States. 2009.

[5] Rajagopalan S. Tuberculosis and aging: a global health problem. Clin Infect Dis 2001; 33(7): 1034-9.

[6] Rajagopalan S, Yoshikawa TT. Tuberculosis in long-term-care facilities. Infect Control Hosp Epidemiol 2000; 21(9): 611-5.

[7] Rajagopalan S, Yoshikawa TT. Tuberculosis in the elderly. Z Gerontol Geriatr 2000; 33(5): 374-80.

[8] Flynn JL, Chan J. Immunology of tuberculosis. Annu Rev Immunol 2001; 19: 93-129.

[9] Cooper AM. Cell-mediated immune responses in tuberculosis. Annu Rev Immunol 2009; 27: 393-422.

[10] Flynn JL. Lessons from experimental Mycobacterium tuberculosis infections. Microbes Infect 2006; 8(4): 1179-88.

[11] Torrelles JB, Schlesinger LS. Diversity in Mycobacterium tuberculosis mannosylated cell wall determinants impacts adaptation to the host. Tuberculosis (Edinb) 2010; 90(2): 84-93.

[12] Stead WW, Lofgren JP, Warren E, Thomas C. Tuberculosis as an endemic and nosocomial infection among the elderly in nursing homes. N Engl J Med 1985; 312(23): 1483-7.

[13] Ijaz K, Dillaha JA, Yang Z, Cave MD, Bates JH. Unrecognized tuberculosis in a nursing home causing death with spread of tuberculosis to the community. J Am Geriatr Soc 2002; 50(7): 1213-8.

[14] CDC. Prevention and control of tuberculosis in facilities providing long-term care to the elderly. Recommendations of the Advisory Committee for Elimination of Tuberculosis. MMWR Recomm Rep 1990; 39(RR-10): 7-13.

[15] Stout RD, Suttles J. Immunosenescence and macrophage functional plasticity: dysregulation of macrophage function by age-associated microenvironmental changes. Immunol Rev 2005; 205: 60-71.

[16] Renshaw M, Rockwell J, Engleman C, Gewirtz A, Katz J, Sambhara S. Cutting edge: impaired Toll-like receptor expression and function in aging. J Immunol 2002; 169(9): 4697-701.

[17] van Duin D, Mohanty S, Thomas V, et al. Age-associated defect in human TLR-1/2 function. J Immunol 2007; 178(2): 970-5.

[18] Rhoades ER, Orme IM. Similar responses by macrophages from young and old mice infected with Mycobacterium tuberculosis. Mech Ageing Dev 1998; 106(1-2): 145-53.

[19] Rottinghaus EK, Vesosky B, Turner J. TLR-2 independent recognition of Mycobacterium tuberculosis by CD11 + pulmonary cells from old mice. Mech Ageing Dev 2010; 131(6): 405-14.

[20] Gonzalez-Juarrero M, Shim TS, Kipnis A, Junqueira-Kipnis AP, Orme IM. Dynamics of macrophage cell populations during murine pulmonary tuberculosis. J Immunol 2003; 171(6): 3128-35.

[21] Brightbill HD, Libraty DH, Krutzik SR, et al. Host defense mechanisms triggered by microbial lipoproteins through toll-like receptors. Science 1999; 285(5428): 732-6.

[22] Donnini A, Argentati K, Mancini R, et al. Phenotype, antigenpresenting capacity, and migration of antigen-presenting cells in young and old age. Exp Gerontol 2002; 37(8-9): 1097-112.

[23] Herrero C, Sebastian C, Marques L, et al. Immunosenescence of macrophages: reduced MHC class II gene expression. Exp Gerontol 2002; 37(2-3): 389-94.

[24] Grubeck-Loebenstein B. Changes in the aging immune system. Biologicals 1997; 25(2): 205-8.

[25] Haynes L, Linton PJ, Eaton SM, Tonkonogy SL, Swain SL. Interleukin 2, but not other common gamma chain-binding cytokines, can reverse the defect in generation of CD4 effector T cells from naive T cells of aged mice. J Exp Med 1999; 190(7): 1013-24.

[26] Haynes L, Eaton SM. The effect of age on the cognate function of CD4+ T cells. Immunol Rev 2005; 205: 220-8.

[27] Maglione PJ, Chan J. How B cells shape the immune response against Mycobacterium tuberculosis. Eur J Immunol 2009; 39(3): 676-86.

[28] Turner J, Frank AA, Orme IM. Old mice express a transient early resistance to pulmonary tuberculosis that is mediated by CD8 T cells. Infect Immun 2002; 70(8): 4628-37.

[29] Cooper AM, Callahan JE, Griffin JP, Roberts AD, Orme IM. Old mice are able to control low-dose aerogenic infections with Mycobacterium tuberculosis. Infect Immun 1995; 63(9): 3259-65.

[30] Turner J, Orme IM. The expression of early resistance to an infection with Mycobacterium tuberculosis by old mice is dependent on IFN type II (IFN-gamma) but not IFN type I. Mech Ageing Dev 2004; 125(1): 1-9. 
[31] Vesosky B, Flaherty DK, Turner J. Th1 cytokines facilitate CD8-Tcell-mediated early resistance to infection with Mycobacterium tuberculosis in old mice. Infect Immun 2006; 74(6): 3314-24.

[32] Berg RE, Cordes CJ, Forman J. Contribution of CD8+ T cells to innate immunity: IFN-gamma secretion induced by IL-12 and IL18. Eur J Immunol 2002; 32(10): 2807-16.

[33] Rottinghaus EK, Vesosky B, Turner J. Interleukin-12 is sufficient to promote antigen-independent interferon-gamma production by CD8 T cells in old mice. Immunology 2009; 128(1 Suppl): e67990.

[34] Vesosky B, Rottinghaus EK, Davis C, Turner J. CD8 T cells in old mice contribute to the innate immune response to Mycobacterium tuberculosis via IL-12p70 dependent and antigen independent production of IFN-\{gamma\}. Infect Immun 2009; 77(8): 3355-63.

[35] Orme IM. Aging and immunity to tuberculosis: increased susceptibility of old mice reflects a decreased capacity to generate mediator T lymphocytes. J Immunol 1987; 138(12): 4414-8.

[36] Orme IM. Aging and immunity to tuberculosis: prolonged survival of old mice infected with Mycobacterium tuberculosis by adoptive immunization with memory-immune T lymphocytes. Cell Immunol 1989; 118(1): 229-33.

[37] Orme IM, Griffin JP, Roberts AD, Ernst DN. Evidence for a defective accumulation of protective T cells in old mice infected with Mycobacterium tuberculosis. Cell Immunol 1993; 147(1): 222-9.

[38] Turner J, Frank AA, Brooks JV, Marietta PM, Vesosky B, Orme IM. Tuberculosis in aged gammadelta $\mathrm{T}$ cell gene disrupted mice. Exp Gerontol 2001; 36(2): 245-54.

[39] Turner J, Orme IM. Identification of altered integrin alpha/beta chain expression on T cells from old mice infected with Mycobacterium tuberculosis. Exp Gerontol 2002; 37(7): 907-16.

[40] Orme IM. The kinetics of emergence and loss of mediator T lymphocytes acquired in response to infection with Mycobacterium tuberculosis. J Immunol 1987; 138(1): 293-8.

[41] Chiu BC, Stolberg VR, Freeman CM, Chensue SW. Mononuclear phagocyte-derived interleukin-10 suppresses the innate pulmonary granuloma cytokine response in aged mice. Am J Pathol 2007; 171(3): 829-37.

[42] Chiu BC, Stolberg VR, Chensue SW. Mononuclear phagocytederived IL-10 suppresses the innate IL-12/IFN-gamma axis in lungchallenged aged mice. J Immunol 2008; 181(5): 3156-66.

[43] Rhoades ER, Frank AA, Orme IM. Progression of chronic pulmonary tuberculosis in mice aerogenically infected with virulent $M y$ cobacterium tuberculosis. Tuber Lung Dis 1997; 78(1): 57-66.

[44] Orme IM. A mouse model of the recrudescence of latent tuberculosis in the elderly. Am Rev Respir Dis 1988; 137(3): 716-8.

[45] Reiley WW, Shafiani S, Wittmer ST, et al. Distinct functions of antigen-specific CD4 T cells during murine Mycobacterium tuberculosis infection. Proc Natl Acad Sci USA 2010; 107(45): 1940813.

[46] Komine-Aizawa S, Yamazaki T, Yamazaki T, et al. Influence of advanced age on Mycobacterium bovis BCG vaccination in guinea pigs aerogenically infected with Mycobacterium tuberculosis. Clin Vaccine Immunol 2010; 17(10): 1500-6.

[47] Silva CL, Bonato VL, Lima VM, Faccioli LH, Leao SC. Characterization of the memory/activated T cells that mediate the long-lived host response against tuberculosis after bacillus Calmette-Guerin or DNA vaccination. Immunology 1999; 97(4): 573-81.

[48] Brooks JV, Frank AA, Keen MA, Bellisle JT, Orme IM. Boosting vaccine for tuberculosis. Infect Immun 2001; 69(4): 2714-7.
[49] Ito T, Takii T, Maruyama M, et al. Effectiveness of BCG vaccination to aged mice. Immun Ageing 2010; 7: 12.

[50] Perez-Guzman C, Vargas MH, Torres-Cruz A, Villarreal-Velarde H. Does aging modify pulmonary tuberculosis? A meta-analytical review. Chest 1999; 116(4): 961-7.

[51] Van den Brande P, Vernies T, Verwerft J, Van Bleyenber R, Vanhoenacker F, Demedts $\mathrm{M}$. Impact of age and radiographic presentation on the presumptive diagnosis of pulmonary tuberculosis. Respir Med 2002; 96(12): 979-83.

[52] Leung CC, Yew WW, Chan CK, et al. Tuberculosis in older people: a retrospective and comparative study from Hong Kong. J Am Geriatr Soc 2002; 50(7): 1219-26.

[53] Wang CS, Chen HC, Yang CJ, et al. The impact of age on the demographic, clinical, radiographic characteristics and treatment outcomes of pulmonary tuberculosis patients in Taiwan. Infection 2008; 36(4): 335-40.

[54] Lee JH, Han DH, Song JW, Chung HS. Diagnostic and therapeutic problems of pulmonary tuberculosis in elderly patients. J Korean Med Sci 2005; 20(5): 784-9.

[55] Morris CD. Pulmonary tuberculosis in the elderly: a different disease? Thorax 1990; 45(12): 912-3.

[56] CDC. Guide to the application of genotyping to tuberculosis prevention and control. Atlanta, GA: US Department of Health and Health Services 2004.

[57] Castle SC, Norman DC, Perls TT, Chang MP, Yoshikawa TT, Makinodan T. Analysis of cutaneous delayed-type hypersensitivity reaction and $\mathrm{T}$ cell proliferative response in elderly nursing home patients: an approach to identifying immunodeficient patients. Gerontology 1990; 36(4): 217-29.

[58] Dorken E, Grzybowski S, Allen EA. Significance of the tuberculin test in the elderly. Chest 1987; 92(2): 237-40.

[59] Mazurek GH, Jereb J, Lobue P, Iademarco MF, Metchock B, Vernon A. Guidelines for using the QuantiFERON-TB Gold test for detecting Mycobacterium tuberculosis infection, United States. MMWR Recomm Rep 2005; 54(RR-15): 49-55.

[60] Kobashi Y, Mouri K, Yagi S, et al. Clinical utility of the QuantiFERON TB-2G test for elderly patients with active tuberculosis. Chest 2008; 133(5): 1196-202.

[61] Leung CC, Yam WC, Yew WW, et al. Comparison of T-Spot.TB and tuberculin skin test among silicotic patients. Eur Respir J 2008; 31(2): 266-72.

[62] CDC. Treatment of tuberculosis. MMWR Morb Mortal Wkly Rep 2003; 53(RR11): 1-77.

[63] Kopanoff DE, Snider DE, Jr., Caras GJ. Isoniazid-related hepatitis: a U.S. Public Health Service cooperative surveillance study. Am Rev Respir Dis 1978; 117(6): 991-1001.

[64] Pande JN, Singh SP, Khilnani GC, Khilnani S, Tandon RK. Risk factors for hepatotoxicity from antituberculosis drugs: a casecontrol study. Thorax 1996; 51(2): 132-6.

[65] Chan CH, Or KK, Cheung W, Woo J. Adverse drug reactions and outcome of elderly patients on antituberculosis chemotherapy with and without rifampicin. J Med 1995; 26(1-2): 43-52.

[66] Mackay AD, Cole RB. The problems of tuberculosis in the elderly. Q J Med 1984; 53(212): 497-510.

[67] Stead WW, To T, Harrison RW, Abraham JH, 3rd. Benefit-risk considerations in preventive treatment for tuberculosis in elderly persons. Ann Intern Med 1987; 107(6): 843-5.

(c) Wang et al.; Licensee Bentham Open.

This is an open access article licensed under the terms of the Creative Commons Attribution Non-Commercial License (http://creativecommons.org/licenses/by-nc/3.0/) which permits unrestricted, non-commercial use, distribution and reproduction in any medium, provided the work is properly cited. 